\title{
Transsphenoidal pituitary surgery effects on obstructive sleep apnea in patients with acromegaly: a systematic review and meta-analysis
}

\begin{abstract}
Context: Acromegaly is a chronic disease associated with excessive growth hormone $(\mathrm{GH})$ and insulin-like growth factor secretion, most commonly as a result of a pituitary adenoma. Patients diagnosed with acromegaly have a high incidence of obstructive sleep apnea. The introduction of endoscopic transnasal transphenoidal pituitary surgery has significantly decreased the risks and morbidities. It remains unclear, however, whether directed pituitary surgery has a significant long-term effect in reducing OSA indices among this population of patients.
\end{abstract}

Objective: To assess whether treatment of acromegaly by means of endoscopic transnasal transphenoidal excision of pituitary adenoma has any significant longterm effects on the hypopnea index and oxygen desaturation index among patients with acromegaly and a co-morbid diagnosis of OSA. Our primary outcome was to evaluate the pre- and post-surgical values for AHI, and our secondary outcomes were the evaluation of GH, ODI, and IGF-1 values.

Methods: In a systematic review and meta-analysis, studies relating to transsphenoidal pituitary adenomectomy in acromegalic patients with obstructive sleep apnea were extracted and analysed based on the inclusion criteria. A search was performed on Scopus, MED-LINE, Pubmed and The Cochrane Library, initially September 3 2015, with an update on October 10, 2015. The effect of the endoscopic transnasal transphenoidal surgery on primary and secondary outcomes was evaluated by metaanalysis.

Results: Three studies (16 patients) were included. The mean age was $46.6 \pm 10.7$ years old and a body mass index (BMI) of $28.7 \pm 2.4 \mathrm{~kg} / \mathrm{m} 2$. Our meta-analysis showed significant surgical treatment effect for AHI $(p=0.0003)$ and IGF-1 $(p<0.0001)$. One study reported GH values, which significantly improved following TSS $(p<0.05)$. No studies included ODI. Each study analysed the results on different amount of time after TSS surgery. Levels of AHI and IGF-1 were significant lower when compared to to the pre-op. same for the conclusion.

Conclusion: The Transsphenoidal surgery could be an option for selected patients to treat acromegaly with good results. Included studies reported an improvement on OSAS following TSS and hormonal control some months after the procedures.

Keywords: acromegaly, sleep apnea, OSA, transsphenoidal surgery
Volume 10 Issue 6 - 2018

\author{
Marcelo Scapuccin, ${ }^{1,2}$ Andrew Thamboo, ${ }^{3}$ \\ Nur Rashid, ${ }^{4}$ Talita Aguiar,' Soroush Zagh,' \\ Stanley Liu,' Paulo Lazarin, ${ }^{2}$ Robson \\ Capassol \\ 'Division of Sleep Surgery, Department of Otolaryngology- \\ Head and Neck Surgery at Stanford University School of \\ Medicine, USA \\ ${ }^{2}$ Department of Otolaryngology-Head and Neck Surgery at \\ Santa Casa School of Medicine, Santa Casa de Misericórida de \\ São Paulo, Brazil \\ ${ }^{3}$ Division of Rhinology, Department of Otolaryngology-Head \\ and Neck Surgery at Stanford University School of Medicine, \\ USA \\ ${ }^{4}$ Department of Otolaryngology-Head and Neck Surgery at \\ University Putra Malaysia, Malaysia
}

\section{Correspondence: Marcelo Scapuccin, Division of Sleep Surgery, Department of Otolaryngology-Head and Neck Surgery at Stanford University School of Medicine, Stanford, CA USA 80I Welch Road, Stanford, CA 94305, USA, Tel 650-723- 565I, Fax 650-723-6685, Email Scapoccin@gmail.com}

Received: October 03, 2017 | Published: November 15, 2018

\section{Introduction}

Obstructive sleep apnea syndrome (OSAS) is characterized by recurrent episodes of partial or complete obstruction of the upper airway during sleep. ${ }^{1}$ In general, OSAS is associated with upper airway abnormalities, including tonsil and adenoid hypertrophy, palatal webbing, nasal obstruction, retrognathia, macroglossia, and laryngeal abnormalities. ${ }^{2,3,4}$ Symptoms like sleepness and laryngopharyngeal reflux are also observed. ${ }^{2,3,4}$ The narrowing of the upper airway, along with the increased airway compliance, allows for the excessive collapsibility of the airway during sleep. (Is there a source for citation here?)

Acromegaly is a disease characterized by the excessive secretion of growth hormone $(\mathrm{GH}){ }^{5}$ This rare condition has an incidence of 5 cases per million per year. ${ }^{6}$ Individuals with acromegaly are at risk of reduced life expectancy due to co-morbidities such as hypertension and diabetes. The incidence of OSAS among patients with acromegaly ranges from $45 \%$ to $80 \%$ of the cases. ${ }^{7}$ Patients with acromegaly present with several characteristics than can contribute to development of sleep disturbances. ${ }^{8}$ The proliferation of soft-tissue in the upper airway contributes to airway narrowing and may lead to partial or complete airway obstruction during sleep, especially at the level of the oropharynx. ${ }^{9}$ Patients with acromegaly who develop OSAS are at the highest risks of morbidity and mortality, as OSAS has been recognized as an independent risk factor for cardiac, metabolic, neurological and peri-operative morbidity. ${ }^{2}$ Rodrigues et al., ${ }^{10}$ reported a prevalence of sleep hypoxemia in $41 \%$ of acromegaly patients, and many agree that the prolonged exposure to low oxygen levels may be the underlying cause for increased mortality rate in those patients.

The majority of patients with acromegaly require multimodal intervention that includes surgery, radiotherapy, and pharmacological therapy with somatostatin analogs and dopamine agonists. ${ }^{5}$ However, the transsphenoidal pituitary surgery remains the main treatment option for $\mathrm{GH}$-secreting tumors. It remains unclear whether the treatment of acromegaly via transsphenoidal pituitary surgery can have long-term beneficial effects in reducing the severity and morbidity of OSAS. Therefore, a systematic review and meta-analysis was undertaken. 


\section{Objectives}

\section{Primary Outcomes}

i. Pre- and post-surgical values for AHI

\section{Secondary Outcomes}

a. Pre- and post-surgical values for GH.

b. Pre- and post-surgical values for ODI.

c. Pre- and post-surgical values for IGF-1.

\section{Methods}

We followed the Preferred Reporting Items for Systematic Reviews and Meta- Analyses (PRISMA) guidelines to develop this systematic review and meta-analysis. ${ }^{11}$

\section{Types of studies}

We reviewed randomized controlled trials, cohort and case control studies that fit the inclusion and exclusion criteria.

\section{Types of participants}

\section{Inclusion criteria}

i. Adult patients (18 years old or older);

ii. Diagnosis of acromegaly and OSAS;

iii. Transsphenoidal surgery (TSS) for the treatment of acromegaly;

iv. Outcome assessment that includes either pre- and post-surgical values for apnea-hypopnea index (AHI), growth hormone (GH), oxygen desaturation index (ODI) and insulin like growth factor 1 (IGF-1).

\section{Exclusion criteria}

a. Pediatric population;

b. Medical management only;

c. Case report studies;

d. Open pituitary surgery;

e. Studies without pre- or post-operative polysomnography (PSG) analysis.

\section{Type of intervention}

TSS is a purely endoscopic approach to the pituitary gland via the sphenoid sinus. It is arguable the most common technique used to surgically remove pituitary tumors.

\section{Search methods for identification of studies}

\section{Electronic searches}

A search was performed on Scopus, MED-LINE, Pubmed and The Cochrane Library, on October 10, 2015. MeSH terms and keywords used for the search included combinations of the following: "acromegaly", "transsphenoidal surgery", "obstructive sleep apnea/ OSA", "pituitary surgery" and "sleep apnea" for the past 10 years (2005-2015). The search strategy was not limited by study design or language.

For each of the searches, the titles and abstracts were screened for relevance followed by a full text review. To minimize the risk of missing relevant articles, we also reviewed the references of each article. Missing data was addressed by contacting original authors of relevant articles.

\section{Data collection, data extraction, and management}

Two reviewers (MS, NR) independently screened the titles and abstracts of studies according to the inclusion/exclusion criteria, read full-text papers, and extracted relevant data using a data extraction form. A third author (SZ) performed a cross-check to ensure accuracy. Reviewers were not blinded in regards to the author, institution, journal of publication, or study results. Any disagreement was discussed between the authors and, if needed, the third reviewer (SZ) helped solving the disagreements. If additional information was required, corresponding authors from the pertinent studies were contacted.

\section{Statistical analysis}

The statistical analyses were performed with JMP Pro 12 (SAS Institute, 2015). The raw data from studies that failed to provide mean and standard deviation were manually input into JMP for calculation. The Review Manager (RevMan) [Computer program] Version 5.3 (Copenhagen: The Nordic Cochrane Centre, The Cochrane Collaboration, 2014) was used for the meta-analysis. When pooling the data in studies, the means, standard deviations, and $95 \%$ confidence intervals (CI) were calculated by REVMAN. Heterogeneity was assessed using the $\chi^{2}$ test and a $\mathrm{p}<0.1$ was considered significant. The $\mathrm{I}^{2}$ test determined the level of heterogeneity $(0 \%$ no heterogeneity, $25 \%$ low, $50 \%$ moderate, $75 \%$ severe). Given the limited number of studies, funnel plots and sensitivity analysis were not performed.

\section{Results}

The search strategy identified 522 abstracts with 32 of them duplicated. A total of 488 titles and abstracts were reviewed. A total of 30 articles were obtained for full text review and 3 articles were eligible for this review. (Figure 1)

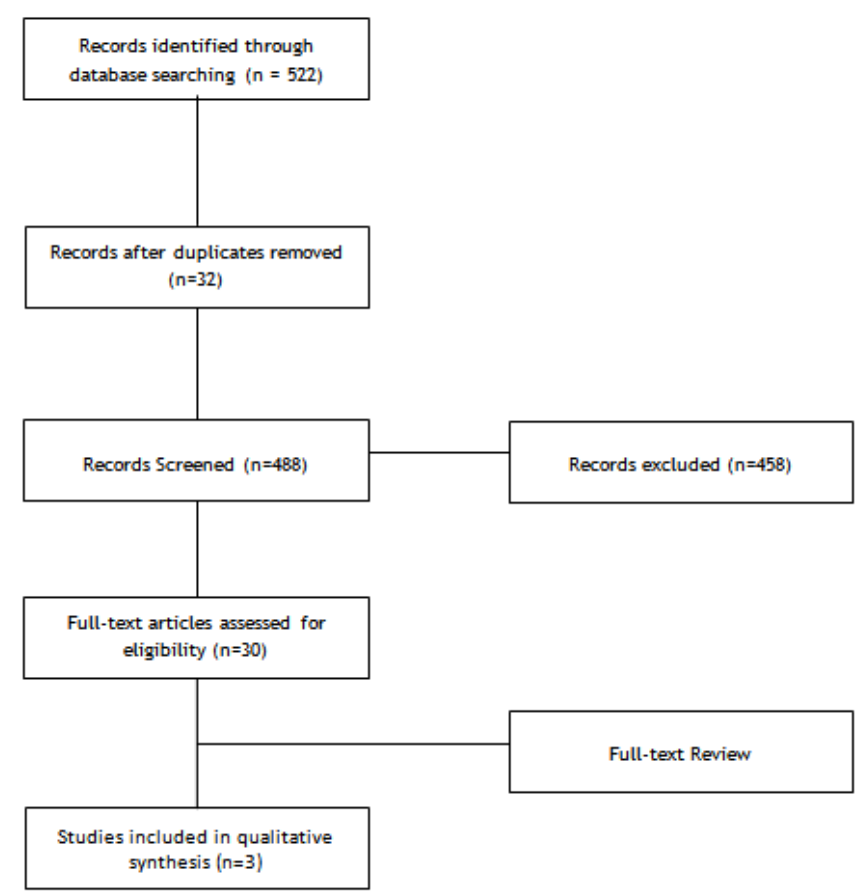

Figure I PRISMA flow diagram for included studies.

\section{Included studies}

Of the 3 studies that were included, there were a total of 16 patients with a mean age of $46.6 \pm 10.7$ years-old and a body mass index (BMI) of $28.7 \pm 2.4 \mathrm{~kg} / \mathrm{m}^{2}$. The BMI values were not reported by Piper et al. ${ }^{12}$ None of the studies reported ODI values. The PSG outcomes for each of the 3 studies are included in Table 1. 
Table I PSG outcomes for pre- and post-TSS in acromegalic patients for included studies

\begin{tabular}{|c|c|c|c|c|c|c|c|c|c|c|}
\hline Study & $\begin{array}{l}\text { Sample } \\
\text { size }\end{array}$ & Age & $\begin{array}{l}\text { BMI }(\mathrm{kg} / \\
\left.\mathrm{m}^{2}\right)\end{array}$ & GH - Pre & $\begin{array}{l}\text { GH - } \\
\text { Post }\end{array}$ & $\begin{array}{l}\text { IGF- } \\
\text { pre }(\mathrm{m} / \\
\mathrm{ml})\end{array}$ & $\begin{array}{l}\text { IGF - } \\
\text { post } \\
(\mathrm{m} / \mathrm{ml})\end{array}$ & $\begin{array}{l}\text { AHI pre- } \\
\text { Op }\end{array}$ & $\begin{array}{l}\text { AHI post- } \\
\text { Op }\end{array}$ & Results \\
\hline $\begin{array}{l}\text { Sze et } \\
\text { al. }{ }^{13}\end{array}$ & 6 & $50.2( \pm 14.4)$ & $29.3( \pm 3.1)$ & $16.8 \pm 16.3$ & $2.0 \pm 1.8$ & $\begin{array}{l}747.7 \pm \\
356.7\end{array}$ & $\begin{array}{l}245.5 \pm \\
41.9\end{array}$ & $4 \mathrm{I}( \pm 20.5)$ & $\operatorname{l}_{( \pm 13.3)}^{1} \cdot 3$ & $\begin{array}{l}\text { After surgery some } \\
\text { patients remain } \\
\text { active, but had a great } \\
\text { improvement on AHI. }\end{array}$ \\
\hline $\begin{array}{l}\text { Piper } \\
\text { et al. }{ }^{12}\end{array}$ & 3 & $50.5( \pm 5,32)$ & - & - & - & $\begin{array}{c}855 \pm \\
257\end{array}$ & $\begin{array}{l}229 \pm \\
206\end{array}$ & $57.9( \pm 31.8)$ & $21.2( \pm 14.0)$ & $\begin{array}{l}50 \% \text { of the study } \\
\text { population got } \\
\text { complete resolution } \\
\text { of sleep apnea. }\end{array}$ \\
\hline
\end{tabular}

Abbreviations: OSAS, obstructive sleep apnea syndrome; AHI, apnea/hypopnea index; PSG, polysomnography; GH, growth hormone; TSS, transsphenoidal surgery

Table 2 Assessment of quality studies for included studies

\section{General characteristics}

Quality assessment of included studies

Quality assessment of cases series studies checklist from National Institute for Health and Clinical Excellence (NICE): (I) Case series collected in more than one center. i.e. multicenter study? (2) Is the hypothesis/aim/objective of the study clearly described? (3) Are the inclusion and exclusion criteria (case definition) clearly reported? (4) Is there a clear definition of the outcomes reported? (5) Were data collected prospectively? (6) Is there an explicit statement that patients were recruited consecutively? (7) Are the main findings of the study clearly described? (8) Are the outcomes stratified? (e.g. disease stage, abnormal test results, patient characteristics); mo. = Months; RCS = Retrospective case series; $\mathrm{PCS}=$ prospective case series

\begin{tabular}{llllllllllll}
\hline Authors, Year & Site & Design & Follow-up & $\mathbf{I}$ & $\mathbf{2}$ & $\mathbf{3}$ & $\mathbf{4}$ & $\mathbf{5}$ & $\mathbf{6}$ & $\mathbf{7}$ & $\mathbf{8}$ \\
\hline Chemla et al. $^{14}$ & France & PCS & 10 mo. & No & Yes & Yes & Yes & Yes & Yes & Yes & Yes \\
Sze et al. ${ }^{13}$ & Switzerland & PCS & 3 mo. & No & Yes & Yes & Yes & Yes & Yes & Yes & Yes \\
\hline
\end{tabular}

There was a variation in the PSG criteria used in the studies. Piper et al., ${ }^{12}$ used the definition proposed by Guilleminault et al in 1978 that patients must experience at least 30 apneic episodes, each lasting a minimum of 10 seconds, to be diagnosed with OSAS. Sze et al., ${ }^{13}$ reported using the American Academy of Sleep Medicine (AASM) scoring criteria but did not specify which year. Chemla et al., ${ }^{14}$ reported using 1999 AASM scoring criteria.

\section{Quality assessment of included papers}

The methodological quality of the included studies was assessed using The National Institute for Health and Clinical Excellence (NICE) quality appraisal tool. ${ }^{15} \mathrm{All}$ of the studies were of high quality and satisfied the majority of the eight items on the NICE qualityassessment tool for case series studies. Of the studies included in this review, one was a retrospective case series and two were prospective in nature.

The main limitation of each case series was the low number of patients enrolled, and that each study was done at a single institution. (Table 2)

\section{Pre- and post-surgical values for AHI}

All studies reported the pre- and post-operative AHI values. Chemla et al. ${ }^{14}$ included 7 patients with OSAS, 4 of which presented with normal PSG parameters following surgery (pre-AHI: 29.0 \pm 16.0 ; post-AHI: $16.0 \pm 10.0, \mathrm{p}>0.05$ ). Sze et al., ${ }^{13}$ reported 6 acromegalic patients with OSAS. Following surgery, the AHI improved significantly for all patients., and none of the patients suffered from OSAS following surgery (pre-AHI: $41.0 \pm 20.5$; post-AHI: $11.3 \pm 13.3$, $\mathrm{p}<0.05)$. Moreover, Sze et al., ${ }^{13}$ noted that OSAS was resolved in all patients irrespective of whether acromegaly was cured or not. Piper et al., ${ }^{13}$ demonstrated a drop in AHI from $57.9 \pm 31.8$ to $22.2 \pm 14.0$ post-operatively in 3 of his patients following transsphenoidal surgery. Additionally, they described that patients noted a subjective improvement of their sleep within 6 to 8 weeks after surgery.

A quantitative analysis was performed pooling the pre-operative and post-operative AHI values from all three studies. By using REvman analysis the difference in pre-operative and post-operative AHI values were found to be statistically significant $(\mathrm{p}=0.0003)$, with low heterogeneity. (Figure 2)

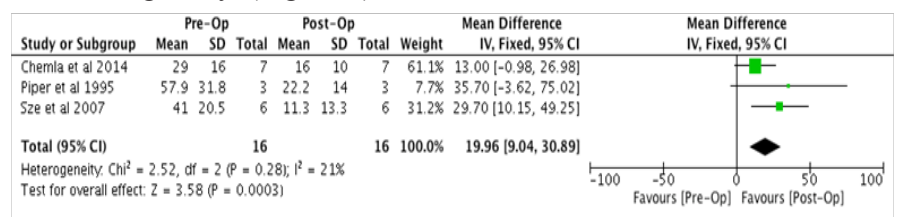

Figure 2 Forest plot of comparison - Pre- and post-operative AHI values following TSS.

\section{Pre- and post-surgical values for GH}

An improvement in GH levels was noted by Sze et al., ${ }^{13}$. There was a statistically significant improvement of the pre-operative $(16.8 \pm 16.3)$ and post-operative (2.0 \pm 1.8$) \mathrm{GH}$ levels of patients suffering from OSAS $(\mathrm{p}<0.05)$. The improvement was also noted in patients without OSAS as their pre-operative scores were $27.0 \pm 19.3$ and post-operative scores 5.7 \pm 5.3 . However, in this group of patients the difference in GH levels was not found to be statistically significant $(\mathrm{p}>005)$. 


\section{Pre- and post-surgical values for IGF-1}

Chemla et al14 reported a dramatic improvement in the IGF-1 levels following tumor resection. The IGF-1 levels improved from $807 \pm 333 \mu \mathrm{g} / \mathrm{L}$ to $207 \pm 69 \mu \mathrm{g} / \mathrm{L}$. This was consistent with the Sze et al. ${ }^{13}$ study where the IGF-1 results changed from $747.7 \pm 356.7 \mu \mathrm{g} / \mathrm{L}$ to $245.5 \pm 41.9 \mu \mathrm{g} / \mathrm{L}(\mathrm{p}<0.05)$. Piper et al., ${ }^{12}$ also noted an improvement in IGF-1 values following surgery from $855 \pm 257 \mu \mathrm{g} / \mathrm{L}$ to $229 \pm 206 \mu \mathrm{g} / \mathrm{L}$.

A quantitative analysis (REVMAN) was performed pooling the pre-operative and post-operative IGF-1 values from all three studies the difference in pre-operative and post-operative IGF-1 values were found to be statistically significant $(\mathrm{p}=0.00001)$, with low heterogeneity. Patients with acromegaly commonly suffer from OSAS as a result of the anatomic alterations caused by the disease. ${ }^{16,17}$ The surgical management of the tumor has relatively high incidences of perioperative and hemodynamic complications. ${ }^{12}$ OSAS is the prevailing form of sleep apnea in patients with acromegaly, and the central and mixed types are less frequent in those cases. ${ }^{16}$ However, some authors have suggested that an abnormal central respiratory control or hormonal mechanisms may also contribute to the development of sleep apnea in patients with acromegaly. Central sleep apnea is associated with the central inhibition of the breathing center likely due to the elevated levels of GH/IGF-1, or to the enhanced somatostatin tone. ${ }^{18}$ The correlation between GH/IGF1 serum levels and severity of sleep apnea is controversial. Grunstein et al., ${ }^{19}$ found a high prevalence of central sleep apnea in patients with acromegaly, and showed that the elevated GH and IGF-I levels potentially contributed to the pathogenesis of central sleep apnea in acromegalic patients. Saeki et al. ${ }^{20}$ found similar results. Both authors found no correlation between GH/IGF-1 and OSAS. However, the hormonal control of GH/IGF-1 levels resulted in the improvement of the most patients sleep problems, providing evidence that controling $\mathrm{GH} / \mathrm{IGF}-1$ levels is essential for all types of sleep apnea in patients with acromegaly. ${ }^{20}$

Only one of the studies included in this analysis discussed GH levels. Sze et al., ${ }^{13}$ concluded that the reduction of GH excess by TSS may cure OSAS and improve insulin resistance and hypertension. They also described that combining the decrease in GH levels with the normalization of the IGF-1 levels led to a significant improvement in AHI values, and resolved the OSAS within a few months. Much like Sze et al., ${ }^{16}$ Chemla et al., ${ }^{14}$ reported that the normalization of the IGF-1 values improved general cardiovascular homeostasis by improving the cardiac autonomic nervous system modulation. Piper et al., ${ }^{12}$ further supported the dramatic improvement in IGF-1 following TSS. Additionally, our meta-analysis of the IGF-1 levels showed a significant $(p<0.00001)$ improvement in IGF-1 following TSS. Despite the controversy of whether IGF-1 plays a role in central sleep apnea or in OSAS, its clearly evident that there is a correlation between the improvement of the sleep apnea followed by TSS and the the decrease in IGF-1 value. Because only one study included the measurements of GH levels, no further analysis was performed in regards to GH levels and TSS.

ODI levels have a strong correlation with other parameters measured during PSG for the diagnose of sleep breathing disorders, and it has the advantage of being measured in portable devices. Although ODI slightly underestimate AHI levels, it has a high accuracy to predict AHI cutoffs. Saeki et al., ${ }^{21}$ was not included on this review because the study did not fit the inclusion criteria; however, this was the only study that reported ODI levels pre- and post-TSS in acromegalic patients. They divided patients into two groups (OSAS group vs nonOSAS) ${ }^{21}$ The difference found in the ODI values between the two groups was $29.1 \pm 15.4$ and $2.5 \pm 1.8$ in the OSAS versus non-OSAS group, respectively. ${ }^{21}$ When comparing preoperative and post-surgery levels, a non-significant improvement in ODI was seen in the OSAS group despite normalization of hormone levels. ${ }^{21}$ In this study, a significant decrease of GH and IGF-1 levels following TSS was also found, with an improvement in most of the sleep complaints. ${ }^{21}$

Although none of the studies included in our review measured ODI levels, all of them measured AHI. In the setting of acromegalic patients, we found a significant improvement in the AHI values following TSS in this review $(\mathrm{p}=0.0003)$. After TSS, the hormonal index tends to decrease, possibly driving important changes in the upper airway anatomy of patients. Sze et al., ${ }^{13}$ reported a beneficial effect of pituitary surgery on upper airway soft tissue. This transformation following the surgery can increase the quality of sleep for acromegaly patients.

One of the reasons behind the difficulty of assessing the impact of TSS on OSAS in acromegaly is the multifactorial pathogenesis of obstructive sleep apnea. OSAS can be related to craniofacial deformations, hypertrophy of the pharyngeal soft tissue, macroglossia and mucosal thickening of the upper airways that increases the hypercollapsibility of posterior and lateral hypopharyngeal walls. ${ }^{22}$

As shown in this review, the literature pertaining to the impact of TSS on OSAS in acromegalic patients is sparse. The findings of this study are based on a limited number of case series, which are, by definition, subject to confounding and study bias. Furthermore, the Piper et al study was descriptive, and represented a small population with potential biases. Moreover, despite the analysis showing low to none heterogeneity in the population studied, it is well understood that the cause for OSAS is multifactorial. Any study compared clinical and surgical treatment for sleep apnea.

Prospective studies with stringent inclusion criteria should be performed to increase the confidence in the correlation between TSS and the improvement of OSAS in acromegalic patients. However, this paper clearly demonstrated that there is evidence showing the improvement in OSAS in the patient population following TSS, and should be considered first line treatment in the management of OSAS for acromegalic patients.

\section{Conclusion}

Sleep apnea syndrome is commonly associated with acromegaly and it should be investigated during their treatment. TSS and hormonal control are associated with the improvement OSAS in patients with acromegaly. Further robust studies are required to further support these findings.

\section{Disclosures}

M.S. is sponsored by the National Council of Technological and Scientific Development (CNPq) program from São Paulo, Brazil.

\section{Acknowledgments}

None.

\section{Conflict of interest}

The author declares there is no conflict of interest.

\section{References}

1. Berry RB, Budhiraja R, Gottlieb DJ, et al. Rules for scoring respiratory events in sleep: Update of the 2007 AASM manual for the scoring of sleep 
and associated events. J Clin Sleep Med. 2012;8(5):597-619.

2. Rita Azeredo Bittencourt L, Martinho Haddad F, Dal Fabbro C, Duma Cintra F, Rios L. General approach in patient with obstructive sleep apnea syndrome. Rev Bras Hipertens. 2009;16:158-163.

3. Xavier SD, Moraes JP, Eckley CA. Prevalence of signs and symptoms of laryngopharyngeal reflux in snorers with suspected obstructive sleep apnea. Braz J Otorhinolaryngol. 2013;79(5):589-593.

4. Young T, Skatrud J, Peppard PE. Risk factors for obstructive sleep apnea in adults. JAMA. 2004;291(16):2013-2016.

5. Chanson P, Salenave S. Acromegaly. Orphanet J Rare Dis. 2008;3:17-18.

6. Lugo G, Pena L, Cordido F. Clinical manifestations and diagnosis of acromegaly. Int J Endocrinol. 2012;10:100-110.

7. Bottini P, Tantucci C. Sleep apnea syndrome in endocrine diseases. Respiration. 2003;70(3):320-327.

8. Mickelson SA, Rosenthal LD, Rock JP, et al. Obstructive sleep apnea syndrome and acromegaly. Otolaryngol-Head Neck Surg. 1994;111(1):2530

9. Isono S, Remmers JE, Tanaka A, et al. Anatomy of pharynx in patients with obstructive sleep apnea and in normal subjects. J Appl Physiol. 1997;82(4):1319-1326.

10. Rodrigues MP, Naves LA, Casulari LA, et al. Using clinical data to predict sleep hypoxemia in patients with acromegaly. Arq Neuropsiquiatr. 2007;65(2A):234-239.

11. Knobloch K, Yoon U, Vogt PM. Preferred reporting items for systematic reviews and meta-analyses (PRISMA) statement and publication bias. $J$ Cranio-Maxillofacial Surg. 2011;39(2):91-92.

12. Piper JG, Dirks BA, Traynelis VC, et al. Perioperative managemen and surgical outcome of the acromegalic patient with sleep apnea
Neurosurgery. 1995;36(1):70-75.

13. Sze L, Schmid C, Bloch KE, et al. Effect of transsphenoidal surgery on sleep apnoea in acromegaly. Eur J Endocrinol. 2007;156(3):321-329.

14. Chemla D, Attal P, Maione L, et al. Impact of successful treatment of acromegaly on overnight heart rate variability and sleep apnea. $J$ Clin Endocrinol Metab. 2014;99(8):2925-2931.

15. Castellani C, Francia G, Dalle et al. Morphological study of upper airways and long-term follow-up of obstructive sleep apnea syndrome in acromegalic patients. Endocrine. 2016;51(2):308-316.

16. Kelly MP. Public health guidance and the role of new NICE. Public Health. 2005;119(11):960-968.

17. Rosenow F, Reuter S, Szelies B, et al. Sleep apnoea in acromegalyprevalence, pathogenesis and therapy. Report on two cases. Presse Med. 1994;23(26):1203-1208.

18. Vasu TS, Grewal R, Doghramji K. Obstructive sleep apnea syndrome and perioperative complications: a systematic review of the literature. J Clin Sleep Med. 2012;8(2):199-207.

19. Grunstein RR, Ho KY, Sullivan CE. Sleep apnea in acromegaly. Ann Intern Med. 1991;115(7):527-532.

20. Saeki N, Isono S, Nishino $\mathrm{T}$, et al. Sleep-disordered breathing in acromegalics-relation of hormonal levels and quantitative sleep study by means of bedside oximeter. Endocrine Journal. 1999;46:585-590.

21. Saeki N, Isono S, Tanaka A, et al. Pre-and post-operative respiratory assessment of acromegalics with sleep apnea--bedside oximetric study for transsphenoidal approach. Endocr J. 2000;47:S61-S64.

22. Herrmann BL, Wessendorf TE, Ajaj W, et al. Effects of octreotide on sleep apnoea and tongue volume (magnetic resonance imaging) in patients with acromegaly. Eur J Endocrinol. 2004;151(3):309-315. 